\title{
Root traits for low input agroecosystems in Africa: lessons from three case studies
}

\author{
Mame Sokhatil Ndoye ${ }^{1}$, Jimmy Burridge ${ }^{2}$, Rahul Bhosale ${ }^{3}$, Alexandre Grondin ${ }^{2}$, and \\ Laurent Laplaze ${ }^{4}$ \\ ${ }^{1}$ CERAAS \\ ${ }^{2}$ Institut de recherche pour le developpement France-Sud \\ ${ }^{3}$ University of Nottingham \\ ${ }^{4}$ Institut de Recherche pour le Développement Centre de Montpellier
}

September 25, 2021

\begin{abstract}
In Africa, agriculture is largely based on low-input and small-holder farming systems that use little inorganic fertilizers and have limited access to irrigation and mechanization. Improving agricultural practices and developing new cultivars adapted to these low-input environments, where production already suffers from climate change, is a major priority for ensuring food security in the future. Root traits improving water and nutrient uptake could represent a solution toward achieving these goals. In this review, we illustrate how breeding for specific root traits could improve crop adaptation and resilience in Africa using three case studies covering very contrasted low-input agroecosystems. First, we review how targeted changes in root system architecture allowed a dramatic increase in common bean yield in low input agroecosystems of South East Africa. We next discuss how root traits could be targeted to improve the productivity and resilience of dryland cereals in the face of climate change and soil degradation. Finally, we evaluate how root traits could be mobilized to develop water-saving rice agroecosystems for West Africa. We conclude with a discussion on how to prioritize target root traits, how they could be validated and made available to breeders and farmers through participatory approaches.
\end{abstract}

\section{Hosted file}

MS_Ndoye_et_al_vf.pdf available at https://authorea.com/users/419386/articles/538823-roottraits-for-low-input-agroecosystems-in-africa-lessons-from-three-case-studies 

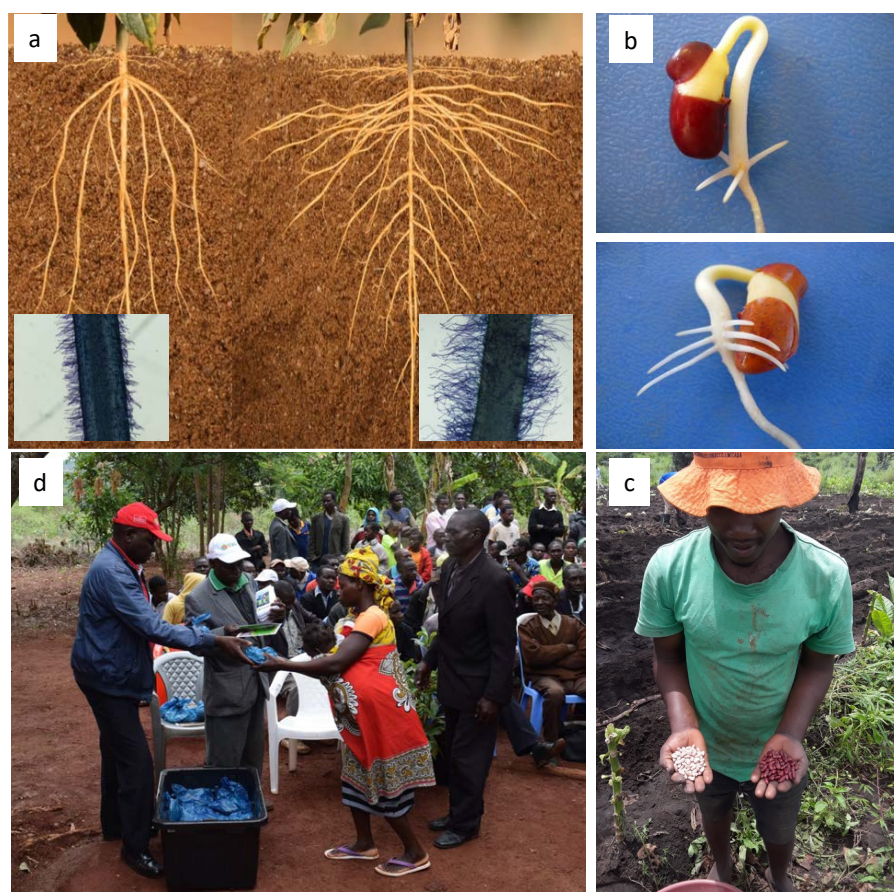

Figure 1. Greater basal root whorl number (BRWN) and longer and denser root hairs were selection targets to improve common bean production for small-holder farmers in Mozambique. a) Root on left shows a typical older variety with fewer basal roots and short root hairs. Image on right represent the improved varieties with more BRWN and longer root hairs. b) Bean seedling 4 days after imbibition showing 1 BRWN (top) and 3 BRWN (bottom). c) Improved beans in hands of smallholder farmers generating certified seed. d) Seed distribution in a Mozambican village as part of a pilot promotional campaign studied seed distribution, adoption and sharing. Image credits: a) still of movie sponsored by the CCRP and Mcknight Foundation (available at roots.psu.edu), photos of long and short root hairs courtesy of Anica Masas, b) courtesy of Katy Barlow, c) photo by Magalhaes Miguel, d) photo of seed distribution by James Burridge. 


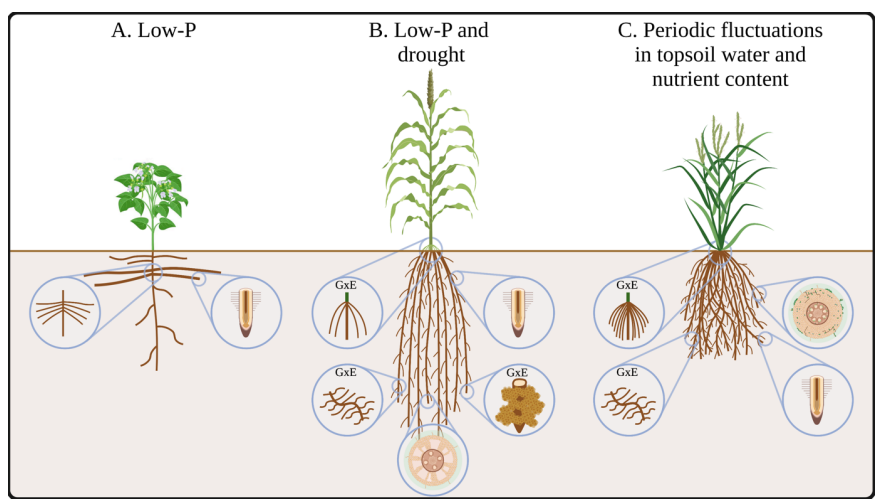

Figure 2: Root traits to optimize resources acquisition in common bean (left), sorghum (middle) and rice (right) when grown in low-input environments. For common bean grown in low-P soils, increased basal root whorl number and longer and denser root hairs were targeted to improve $P$ acquisition efficiency. Proposed selection targets for sorghum grown in droughtprone regions with low-P soils in Sahelian Africa include greater root hair length and density for increased $\mathrm{P}$ acquisition, combined with more aerenchyma for decreased carbon cost of root tissues and potentially reduced xylem vessel diameter for increased water use efficiency. Plasticity (GXE) in crown root number and lateral root branching at depth as well as rhizosheath formation are potential drought adaptive responses. In alternate wet and dry rice agroecosystems, proposed adaptive responses to periodic topsoil fluctuations in water and nutrient content include root hair development and plasticity in crown root number and topsoil lateral root branching (increased upon dry-down), combined with greater beneficial interactions with arbuscular-mycorrhizal fungi. Figure was created with BioRender.com. 